\title{
ON THE MECHANISM OF THE FORMATION OF MAGNETO- HYDRODYNAMIC VORTICES IN THE SOLAR PLASMA
}

\author{
E. A. Pashitskii \\ Institute of Physics, National Academy of Sciences of Ukraine, \\ pr. Nauki 46, Kyiv, 03680 Ukraine, pashitsk@iop.kiev.ua
}

\begin{abstract}
Based on the magnetohydrodynamic (MHD) equations for an incompressible conductive viscous fluid, the possible mechanism of the formation of giant MHD vortices recently discovered in the solar atmosphere (chromosphere) is analyzed. It is assumed that these vortices arise in the regions of the solar surface (photosphere) with ascending flows of hot plasma that arrives from the inner regions of the Sun as a result of thermal convection and is accelerated upward under the action of the chromospheric plasma pressure gradient. It is shown that, under the assumption of plasma incompressibility and flow continuity, the ascending plasma flows induce converging radial plasma flows, which create the convective and Coriolis nonlinear hydrodynamic forces due to the nonzero initial vorticity of the chromospheric plasma caused by Sun's rotation. The combined action of these two forces leads to the exponential acceleration of the solid-body rotation of plasma inside the ascending flow, thereby creating a vortex that generates an axial magnetic field, in agreement with astrophysical observations.
\end{abstract}

Keywords: solar plasma, MHD vortex

\section{Introduction}

Recent publication [1] reported observation of cylindrically symmetric vortex structures with characteristic radii of $R \approx 500 \mathrm{~km}$ in the solar atmosphere in the UV spectral region. There may be more than $10^{4}$ such structures simultaneously on the Sun's surface. These vortices penetrate through the entire chromosphere reaching the lower layers of the solar corona at an altitude of $2500 \mathrm{~km}$. The existence of such magnetohydrodynamic (MHD) vortices, which were earlier predicted in [2-4], is now confirmed by means of precision optical measurements of the Doppler shifts of the absorption lines of iron, calcium, and helium ions [5] corresponding to the vortex motion of the chromospheric plasma with velocities on the order of $4 \mathrm{~km} / \mathrm{s}$. It was suggested in [1] that such MHD vortices could be responsible for the heating of the corona plasma up to temperatures of several million degrees due to dissipation of the energy of Alfvén [6-10] and magnetosonic [11] waves excited by the vortex plasma motion. In [1], vortex structures were simulated numerically by using the MHD equations for a perfectly conducting ideal incompressible fluid with allowance for the processes of radiative energy transfer. It was assumed in [1] that an MHD vortex is accelerated due to its radial compression by con- verging plasma flows, provided that the initial angular momentum of the vortex is conserved.

However, as will be shown below, an MHD vortex in plasma cannot be regarded as a conservative system in which the angular momentum is constant, because there is a permanent influx of matter with a nonzero vorticity into the vortex core from the surrounding chromospheric plasma, i.e., such a vortex is an open nonequilibrium system. As a result, the mechanism of the formation and evolution of an MHD vortex in the solar chromosphere cannot be considered completely established. In the present work, we analyze a possible mechanism of the formation of an MHD vortex in the solar chromosphere and the accompanying generation of magnetic fields and ohmic heating of the chromospheric plasma in the framework of an approximate description based on the MHD equations for an incompressible viscous fluid with a finite conductivity [12]. It is assumed that such vortices are initiated by the ascending flows of hot plasma that arise in some "hot spots" on the Sun's surface, i.e., in the regions of the photosphere where hot plasma rises from the inner regions of the Sun as a result of convection. This hot plasma expands and rapidly flows upwards in the gravitational field through the surrounding colder chromospheric plasma, whose pressure rapidly drops with altitude. Under the conditions of the flow continuity and plasma incompressibility, the ascending vertical flow creates converging radial flows. At a nonzero initial vorticity of the chromospheric plasma caused by the Sun's rotation, such converging flows give rise to convective and Coriolis nonlinear hydrodynamic forces. The combined action of these two forces leads to the acceleration of the solid-body rotation of plasma in the core of the MHD vortex, similar to the mechanism of the formation of air vortices in the Earth's atmosphere (such as whirlwinds, tornadoes, and typhoons) earlier analyzed in [13]. Here, we analyze axisymmetric vortex solutions to the nonlinear MHD equations with separable variables that satisfy the continuity equation and cause the kinematic and magnetic viscosities of an incompressible conductive fluid to vanish. Such solutions satisfy the principle of the minimum entropy generation in an open nonequilibrium system [13], i.e., correspond to the minimum dissipation rate of the kinetic and magnetic energies of the MHD vortex. It is shown that the vortex state is characterized by the exponential growth of both the azimuthal rotation velocity of the MHD vortex and the axial magnetic field, which qualitatively agrees with the observations $[14,15]$ of the local concentration of the magnetic field under vortex motion of the solar plasma. 
Such a growing magnetic field generates an azimuthal electric current in the external shell of the MHD vortex, which should lead to the ohmic heating of plasma in the chromosphere and lower layers of the solar corona. It is shown that, due to the instability of the growing tangential discontinuity of the azimuthal velocity at the boundary of the vortex core, strong local turbulence with an anomalously high viscosity develops in the surface layer, which leads to the dissipation of MHD vortices.

\section{Basic equations for the description of MHD vor- tices in the solar chromosphere}

To describe MHD vortices in the solar chromosphere, we will use the well-known set of MHD equations for an incompressible viscous conductive fluid [14].

We note that the MHD approximation can be used to describe electron--ion plasma only if the cyclotron radii of ions and electrons, as well as their Debye screening lengths and their free path lengths along the magnetic field, are smaller than the characteristic spatial scales of the problem (in particular, the MHD vortex dimensions). Such an approximation can also be used to describe a weakly ionized plasma with a high particle collision frequency and low electric conductivity. At the same time, plasma in a magnetic field can be assumed to be incompressible if the velocity of its macroscopic motion is lower than both the adiabatic sound speed $c_{s}=\sqrt{\Gamma P / \rho}$ (where $\Gamma$ is the adiabatic index) and the Alfvén velocity $c_{A}=H / \sqrt{4 \pi \rho}$.

Let us analyze cylindrical axisymmetric (i.e., independent of the azimuthal angle $\varphi$ ) vortex flows of a conductive fluid (plasma) in which the self-consistent magnetic field has only the azimuthal and axial components, $\mathbf{H}\left(0, H_{\varphi}, H_{z}\right)$. In this case, the set of MHD equations in cylindrical coordinates with allowance for the gravity acceleration $g$ (which is directed vertically downward, i.e., in negative $z$ direction) takes the form

$$
\begin{gathered}
\frac{\partial v_{r}}{\partial t}+v_{r} \frac{\partial v_{r}}{\partial r}+v_{z} \frac{\partial v_{r}}{\partial z}-\frac{v_{\varphi}^{2}}{r} \\
=-\frac{1}{\rho} \frac{\partial}{\partial r}\left(P+\frac{H_{\varphi}^{2}+H_{z}^{2}}{8 \pi}\right)+\frac{H_{\varphi}^{2}}{4 \pi \rho r}+v \cdot \Delta v_{r}, \\
\frac{\partial v_{\varphi}}{\partial t}+v_{r} \frac{\partial v_{\varphi}}{\partial r}+v_{z} \frac{\partial v_{\varphi}}{\partial z}+\frac{v_{r} v_{\varphi}}{r} \\
=\frac{1}{4 \pi \rho} H_{z} \frac{\partial H_{\varphi}}{\partial z}+v \cdot \Delta v_{\varphi}, \\
\frac{\partial v_{z}}{\partial t}+v_{r} \frac{\partial v_{z}}{\partial r}+v_{z} \frac{\partial v_{z}}{\partial z}=-\frac{1}{\rho} \frac{\partial}{\partial z}\left(P+\frac{H^{2}}{8 \pi}\right) \\
-g+\frac{1}{4 \pi \rho} H_{z} \frac{\partial H_{z}}{\partial z}+v \cdot \Delta v_{z}, \\
\frac{\partial H_{\varphi}}{\partial t}+v_{r} \frac{\partial H_{\varphi}}{\partial r}+v_{z} \frac{\partial H_{\varphi}}{\partial z} \\
=H_{z} \frac{\partial v_{\varphi}}{\partial z}+\frac{v_{r} H_{\varphi}}{r}+v_{m} \cdot \Delta H_{\varphi},
\end{gathered}
$$

$$
\frac{\partial H_{z}}{\partial t}+v_{r} \frac{\partial H_{z}}{\partial r}+v_{z} \frac{\partial H_{z}}{\partial z}=H_{z} \frac{\partial v_{z}}{\partial z}+v_{m} \cdot \Delta H_{z},
$$

where $v_{r}, v_{\varphi}$, and $v_{z}$ are the radial, azimuthal, and axial components of the hydrodynamic velocity of the fluid, respectively, $v$ and $v_{m}$ are the kinematic and magnetic viscosities, and the Laplace operator is

$$
\Delta=\frac{\partial^{2}}{\partial r^{2}}+\frac{1}{r} \frac{\partial}{\partial r}+\frac{\partial^{2}}{\partial z^{2}} .
$$

We note that terms $v_{\varphi}^{2} / r$ and $v_{r} v_{\varphi} / r$ on the left-hand side of equations (1) and (2) describe the centrifugal force and the local Coriolis nonlinear hydrodynamic force, respectively.

In this case, the continuity equation for an incompressible fluid, $\operatorname{div} v=0$, and Maxwell's equation for the solenoidal magnetic field, $\operatorname{div} \mathbf{H}=0$, take the form

$$
\frac{\partial v_{r}}{\partial r}+\frac{v_{r}}{r}+\frac{\partial v_{z}}{\partial z}=0 ; \quad \frac{\partial H_{z}}{\partial z}=0 \text {. }
$$

The simplest formal solutions to Eqs. (7) with the separable variables $r$ and $z$ in the region $r \leq R_{0}$ have the form

$$
\begin{gathered}
v_{r}(r)=-\beta \cdot r ; \quad v_{z}(z)=v_{z 0}+\alpha \cdot z ; \\
H_{z}=h=\text { const. }
\end{gathered}
$$

where the parameters $\alpha$ and $\beta$ are related by the formula

$$
(\alpha-2 \beta)=0 \text {. }
$$

\section{Ascending flows of plasma in solar chromosphere}

The expression for the radial velocity $v_{r}(r)$ in Eq. (8) describes an incompressible radial plasma flow converging toward the axis that is induced by the ascending plasma flow the axial velocity of which increases linearly along $z$ axis, $v_{z}(z)=v_{z 0}+\alpha z$. According to timeindependent equation (3),

$$
v_{z} \frac{\partial v_{z}}{\partial z}=-\frac{1}{\rho} \frac{\partial P}{\partial z}-g+v \frac{\partial^{2} v_{z}}{\partial z^{2}}
$$

such a velocity can appear under the action of the pressure $P$, which decreases with altitude according to the square law

$$
P(z)=P_{0}-\rho\left(a z+b z^{2}\right),
$$

where $a=\left(\alpha v_{z 0}+g\right)$ and $b=\alpha^{2} / 2$. As will be shown below, such a dependence of the pressure on $z$ can occur in the gravitational field at relatively low altitudes, provided that the plasma temperature sufficiently fast drops with altitude.

Let us suppose that hot plasma in a certain region of radius $R_{0}$ in the solar photosphere flows up due to thermal convection in the gravitational field. This plasma expands and flows upward under the action of the buoyancy force in the colder denser chromospheric plasma. As the plasma flows up and expands, it plasma cools down, so that its temperature in the initial stage decreases almost linearly with altitude,

$$
T(z) \approx T_{0} \cdot(1-\chi z) ; \quad \chi=-\left(\frac{\partial \ln T}{\partial z}\right)_{z=0}>0 .
$$


In this case, to within second-order terms in $z$, the barometric formula for the pressure of the chromospheric plasma in the Sun's gravitational field takes the form

$$
\begin{gathered}
P(z)=P_{0} \cdot \exp \left\{-\frac{m g z}{k_{B} T(z)}\right\} \\
\approx P_{0} \cdot\left[1-\frac{m g z}{k_{B} T_{0}}-\frac{m g}{k_{B} T_{0}}\left(\chi-\frac{m g}{2 k_{B} T_{0}}\right) z^{2}\right] .
\end{gathered}
$$

For $\chi>m g / 2 k_{B} T_{0}$, this formula coincides with the adopted dependence (11) of the pressure on the $z$ coordinate if we set $a=P_{0} \cdot m g / k_{B} T_{0} \quad$ and $b=\left(\chi-m g / 2 k_{B} T_{0}\right) \cdot a$ (where $m$ is the mass of a hydrogen atom). As a result, we obtain two equations for determining two parameters, $v_{z 0}$ and $\alpha$, entering into the expression for the increasing velocity of the ascending flow,

$$
\begin{aligned}
& \alpha v_{z 0}=g \cdot\left(\frac{c_{s}^{2}}{\Gamma} \frac{m}{k_{B} T_{0}}-1\right)>0, \\
& \alpha^{2}=\frac{m g}{k_{B} T_{0}} \cdot\left(2 \chi-\frac{m g}{k_{B} T_{0}}\right)>0 .
\end{aligned}
$$

Taking into account the parameters of plasma in the lower layers of the solar chromosphere $\left(c_{s} \approx 10 \mathrm{~km} / \mathrm{s}\right.$ and $\left.T_{0} \approx 6000 \mathrm{~K}\right)$ and the value of the gravity acceleration on the Sun's surface $\left(g \approx 274 \mathrm{~m} / \mathrm{s}^{2}\right)$, we obtain $\alpha v_{z 0} \approx 57.5 \mathrm{~m} / \mathrm{s}^{2}$ and $m g / k_{B} T_{0} \approx 5.5 \cdot 10^{-6} \mathrm{~m}^{-1}$. However, in this case, the parameter $\chi$, which has the dimension of the reciprocal length and characterizes the cooling rate of hot plasma with altitude, remains undefined, due to which the values of $\alpha$ and $v_{z 0}$ cannot be estimated independently (see below).

Note that the radius $R_{0}$ of the ascending flow should increase with altitude, which prevents separation of the variables $r$ and $z$ in the MHD equations. However, if the longitudinal inhomogeneity scale $L$ of the plasma flow radius is much larger than $R_{0}$, then, with a high degree of precision, we can set $R_{0}=$ const, which substantially simplifies the problem and allows us to investigate the main physical processes affecting the dynamics and evolution of MHD vortices in the solar atmosphere by means of an approximate procedure of separation of variables without recourse to complicated computer simulations.

\section{Solid-body vortex rotation of plasma}

Let's assume that at $r \leq R_{0}$ the azimuthal components of the velocity and magnetic field are independent of $z$ and have the form

$$
v_{\varphi}(r)=\omega \cdot r ; \quad H_{\varphi}(r)=\gamma \cdot r .
$$

After the substitution of expressions (8) and (15) into Eqs. (1)-(5), a large number of terms on the right-hand sides (including those containing Laplace operator (6)) vanish. This corresponds to the zero kinematic and magnetic viscosities of the incompressible conductive fluid (plasma), i.e., in fact to the nondissipative solid-body rotation of the MHD vortex core at $r<R_{0}$.

Substituting Eqs. (8) and (15) into Eqs. (1), (2), (4), and (5) and assuming that the parameters $\gamma, \omega$ and $h$ are functions of $t$, we obtain the following set of first-order equations that describe the dynamics of the MHD vortex core:

$$
\begin{gathered}
\omega^{2}(t)-\beta^{2}=\frac{1}{r \rho} \cdot \frac{\partial P}{\partial r}+\frac{\gamma^{2}(t)}{2 \pi \rho}, \\
\frac{d \omega}{d t}-2 \beta \cdot \omega(t)=0 \\
\frac{d h}{d t}-\alpha \cdot h(t)=0 .
\end{gathered}
$$

In this case, Eq. (4) is reduced to the condition $d \gamma / d t=0$, which is a consequence of the mutual compensation of the nonlinear terms $v_{r} \partial H_{\varphi} / \partial r$ and $v_{r} H_{\varphi} / r$ on the right- and left-hand sides of Eq. (4). This allows us to neglect the azimuthal component of the self-consistent magnetic field $H_{\varphi}$ in the MHD vortex.

Since, according to Eq. (9), we have $\alpha=2 \beta$, solutions to Eqs. (17) and (18) have the form

$$
\omega(t)=\omega(0) \cdot e^{\alpha t}, \quad h(t)=h(0) \cdot e^{\alpha t},
$$

where $\omega(0)$ and $h(0)$ are the initial values of the angular plasma rotation velocity and axial magnetic field, respectively.

Equation (16) governs spatial distribution and temporal behavior of plasma pressure in the core of the MHD vortex, which has the following form in the cyclostrophic regime of rotation of an incompressible fluid:

$$
\begin{aligned}
P(r, z, t)= & \tilde{P}_{0}+\frac{\rho r^{2}}{2}\left[\omega^{2}(t)+\omega^{2}(0)-\beta^{2}\right] \\
& -\frac{\rho z^{2}}{2} \alpha^{2}(t)-\rho z \tilde{g},
\end{aligned}
$$

where $\tilde{P}_{0}$ is the pressure at the vortex axis (see below), and $\tilde{g}=g+v_{z 0} \alpha$.

\section{Exponential regime of MHD vortex evolution}

It follows from Eq. (19) that the angular velocity of the solid-body rotation of the MHD vortex core and the axial component of the self-consistent magnetic field increase exponentially with a characteristic time of $t_{\text {exp }}=1 / \alpha$.

We see that the source of the exponential acceleration of the vortex core rotation is the linear increase in the axial velocity of the ascending flow of incompressible plasma, $v_{z}(z)=\left(v_{z 0}+\alpha z\right)$, as it propagates upward in the gravitational field of the Sun through the solar chromosphere, in which the temperature and pressure decrease with increasing altitude.

The value of $\alpha$ can be estimated by equating the velocity of the ascending flow $v_{z}(z)=v_{z 0}+\alpha z$ to the speed of sound at the upper boundary of the solar chromosphere, 
$L \approx 2500 \mathrm{~km}$. In the rarefied upper layers of the chromospheric plasma, the speed of sound is $\tilde{c}_{s}=\sqrt{2 k_{B} \tilde{T} / m}$, where $\tilde{T} \approx 3 \cdot 10^{4} \mathrm{~K}$, so that $\tilde{c}_{s} \approx 20 \mathrm{~km} / \mathrm{s}$. Assuming that $v_{z 0} \leq \tilde{c}_{s}$, we find that $\alpha \leq \tilde{c}_{s} / L \approx 8 \cdot 10^{-3} \mathrm{~s}^{-1}$ and the characteristic time of the exponential evolution of the MHD vortex is $t_{\text {exp }} \geq L / \tilde{c}_{s} \approx 2 \mathrm{~min}$.

It should be noted that the exponential growth of the axial magnetic field $H_{z}(t)=h(t)$ qualitatively agrees with the local concentration of the magnetic field during the vortex motion of the chromospheric plasma observed in $[14,15]$. In this case, the local Alfvern velocity $c_{A}(t)=H_{z}(t) / \sqrt{4 \pi \rho}$ inside the vortex core grows exponentially in time (see (19)).

According to expression (20), the plasma pressure on the vortex axis at $z=0$ in the cyclostrophic regime of vortex rotation is

$$
\tilde{P}_{0}(0, t) \approx P_{0}-\frac{\rho R_{0}^{2}}{2} \cdot \omega^{2}(t) .
$$

According to Eqs. (19) and (21), this pressure decreases exponentially in time and vanishes at the time

$$
t_{0}=\frac{1}{2 \alpha} \cdot \ln \left[\frac{2 P_{0}}{\rho R_{0}^{2} \cdot \omega^{2}(0)}\right] \approx \frac{1}{\alpha} \cdot \ln \left(c_{s} / V_{0}\right),
$$

where $V_{0}=R_{0} \cdot \omega(0)$ is the initial azimuthal velocity of the vortex motion of plasma $\left(V_{0} \ll c_{s}\right)$. Since the negative pressure in the system leads to instability (collapse), an MHD vortex cannot exist at $t>t_{0}$. The time $t_{0}$ can be approximately estimated if we assume that, in the order of magnitude, the initial value of the angular velocity $\omega(0)$ coincides with the average solar atmosphere vorticity caused by the nonuniform (liquid-like) rotation of the Sun's surface with the angular velocity varying with latitude from $\Omega \approx 1 / 28$ day on the equator to $\Omega \approx 1 / 33$ day at a latitude of $75^{\circ}$. Estimates show that the initial vorticity is $\omega(0) \approx 2 \cdot 10^{-6} \mathrm{~s}^{-1}$, which is one order of magnitude higher than the vorticity caused by the global Coriolis force on the solar surface.

As a result, the initial azimuthal velocity of plasma at the boundary of a vortex of radius $R_{0} \approx 500 \mathrm{~km}$ is $V_{0} \approx 1 \mathrm{~m} / \mathrm{s}$. Taking into account that the density of the chromospheric plasma on the Sun's surface is on the order of $\rho \approx 5 \cdot 10^{-9} \mathrm{~g} / \mathrm{cm}^{3}$ and the temperature is $T \approx 6000 \mathrm{~K}$ and assuming that the density of hydrogen atoms is $n \approx 3 \cdot 10^{15} \mathrm{~cm}^{-3}$, we find using the approximation of an ideal gas that the speed of sound is $c_{s} \approx 10 \mathrm{~km} / \mathrm{s}$. Hence, with allowance for the above estimate of $\alpha$ and disregarding dissipation of the vortex kinetic energy (see below), we find that the maximum time of the exponential evolution of an MHD vortex is $t_{0} \approx 15 / \alpha \approx 30 \mathrm{~min}$.

On the other hand, it should be taken into account that the solid-body rotation of the vortex core begins to decelerate when the azimuthal velocity at $r=R_{0}$ becomes compara- ble with the speed of sound, because, at such velocities, the compressibility effects come into play and the fluid (plasma) acquires a nonzero bulk viscosity. Therefore, taking into account the exponential growth of the rotation velocity of the vortex core, the maximum azimuthal velocity $v_{\varphi}^{\max }\left(R_{0}, t_{s}\right)=V_{0} \cdot \exp \left(\alpha \cdot t_{s}\right) \approx c_{s}$ is reached over a time $t_{s}=1 / \alpha \cdot \ln \left(c_{s} / V_{0}\right)$, which coincides with $t_{0}$. We note that the velocity of the vortex motion of the chromospheric plasma determined from the Doppler shift of the spectral lines is $4 \mathrm{~km} / \mathrm{s}$, which is half as large as the speed of sound, whereas according to computer simulations, the vortex velocity at altitudes of $2500 \mathrm{~km}$, where the speed of sound is $c_{s} \approx 20 \mathrm{~km} / \mathrm{s}$, can reach $15 \mathrm{~km} / \mathrm{s}$.

The time $t_{0} \approx t_{s}$ also determines the maximum values of the axial component of the magnetic field inside the vortex core, $H_{z}=h(0) \cdot \exp \left(\alpha t_{0}\right)$, and the corresponding local

Alfvén velocity $c_{A}^{\max }=\sqrt{h^{2}(0) / 4 \pi \rho} \cdot \exp \left(\alpha t_{0}\right)$. Assuming that the factor in front of the exponential in the last expression is on the order of the initial vortex velocity $V_{0} \approx 1 \mathrm{~m} / \mathrm{s}$, we find that $c_{A}^{\max } \approx 10 \mathrm{~km} / \mathrm{s}$. In this case, the magnetic field reaches its maximum value of $H_{z}^{\max } \approx 250 \mathrm{G}$, which agrees with observational data $[14,15]$.

Note that the excitation of Alfvén and magnetosonic waves in the MHD vortex core cannot lead to vortex deceleration and plasma heating, because the Alfvén velocity grows according to the same exponential law as the azimuthal velocity of plasma rotation, whereas the speed of sound decreases due to a decrease in the pressure in the vortex core.

\section{Instability of the tangential discontinuity of the azimuthal velocity and local turbulence on the sur- face of the MHD vortex core}

The exponential growth of the angular velocity of the solid-body plasma rotation in the MHD vortex core, where the radial and axial velocities are directly proportional to the radius $r$, is caused, as was mentioned above, by the combined action of the convective and Coriolis nonlinear hydrodynamic forces, which are equal in magnitude. However, in the external region $r \geq R_{0}$, where the radial and azimuthal velocities are proportional to $r^{-1}$, these forces, as can be easily verified, have opposite signs and exactly balance one other. As a result, there is no acceleration of the initial vorticity $\omega(0)$. Therefore, the azimuthal velocity experiences an exponentially growing jump (tangential discontinuity) at the vortex core boundary.

As was shown for the first time in [13], the exponential growth of the velocity jump leads to the absolute instability of surface perturbations accompanied by the growth of their amplitude according to the double exponential law of the form

$$
\left|\xi_{k}(t)\right|=\left|\xi_{k}(0)\right| \cdot \exp \left\{\frac{k V_{0}}{2 \alpha} \cdot\left(e^{\alpha t}-\alpha t\right)\right\},
$$

where $\xi_{k}(0)$ is the initial perturbation with the wavenumber $k$ at $t=0$. 
Such instability develops much faster than the exponential acceleration of the solid-body rotation of the MHD vortex core. As a result, a strongly turbulent state is rapidly established near the vortex surface. Turbulence is localized in a layer of thickness $\delta$, which is comparable with the maximum amplitude of turbulent pulsations $l^{*}=\left|\xi_{k}\right|_{\max }$. The amplitude reaches its maximum value $\left|\xi_{k}\right|_{\max } \equiv\left|\xi_{k}\left(t_{\max }\right)\right|$ at the time $t_{\max }$ at which the velocity of turbulent pulsations

$$
\tilde{v}_{k}(t) \equiv \frac{d \xi_{k}}{d t}=\left|\xi_{k}(t)\right| \cdot \frac{k V_{0}}{2} \cdot(\exp \{\alpha t\}-1)
$$

becomes equal to the speed of sound $c_{s}$ and the effects of compressibility and finite viscosity come into play. It is well known that the wavenumber $k$ of the most unstable surface perturbations has the same order of magnitude as the reciprocal thickness of the transition layer $1 / \delta$. Hence, we have

$$
l^{*} \approx \delta \approx\left|\xi_{k}\right|_{\max } \approx \pi / k_{\max } .
$$

At $\alpha t_{\max } \gg 1$, expression (24) yields

$$
t_{\max } \approx \frac{1}{\alpha} \cdot \ln \left(\frac{c_{s}}{\pi V_{0}}\right) \leq t_{0} .
$$

Thus, the peak amplitude of turbulent pulsations cannot exceed

$$
\left|\xi_{k}\right|_{\max }=\xi_{0} \cdot \exp \left\{\frac{k V_{0}}{2 \alpha} \cdot \exp \left(\alpha t_{0}\right)\right\} \text {. }
$$

Taking the logarithm of expression (27) with allowance for relationships (23) and (25), we obtain to within logarithmic accuracy the following transcendental equation for the characteristic scale length of turbulent pulsations $l^{*}$ and, accordingly, the effective width $\delta$ of the turbulent transition layer on the vortex core boundary:

$$
\delta \approx \frac{c_{s}}{2 \alpha \cdot \ln \left(\delta / \xi_{0}\right)} \approx \frac{600}{\ln \left(\delta / \xi_{0}\right)} \mathrm{km} .
$$

Assuming that the amplitude $\xi_{0}$ of the initial fluctuations of quasineutral plasma is on the order of the Debye screening length $D=\sqrt{k_{B} T / 8 \pi e^{2} n_{e}}$, which, at a chromospheric plasma temperature of $T \approx 6000 \mathrm{~K}$ and an electron density of $n_{e} \approx 3 \cdot 10^{15} \mathrm{~cm}^{-3}$, is $D \approx 10^{-5} \mathrm{~cm}$, we find that the thickness of the turbulent region surrounding an MHD vortex is $\delta \approx 25 \mathrm{~km}$, which corresponds to $\ln (\delta / D) \approx 25$. If the amplitude of turbulent pulsations is known, then, applying the dimensionality analysis, we find that the turbulent viscosity of plasma inside the turbulent layer is $v^{*} \approx c_{s} \cdot l^{*} / 3 \approx 8 \cdot 10^{7} \mathrm{~m}^{2} / \mathrm{s}$.

The anomalously large effective viscosity of the turbulent layer improves the stability of the MHD vortex core during its evolution. In this regard, it may be supposed that the observed filamentary structure of strong solar flares is caused by the simultaneous formation of a large number of MHD vortices in the photospheric hot spots.

\section{Deceleration of MHD vortices due to viscous en- ergy dissipation and ohmic losses in the turbulent layer}

As we mentioned above, solutions with separable variables correspond to zero kinematic and magnetic viscosities of the incompressible conductive fluid (plasma) both inside the MHD vortex core at $r \leq R_{0}$ and in the external region $r \geq\left(R_{0}+\delta\right)$. However, the kinetic energy of the vortex should dissipate inside the turbulent surface layer of thickness $\delta \ll R_{0}$ with an anomalously large turbulent viscosity $v^{*}$. The energy dissipation rate per unit vortex length is determined by the relationship

$$
\frac{d E_{k i n}^{*}}{d t}=-\frac{\rho v^{*}}{2} \int d V\left(\frac{\partial v_{\varphi}}{\partial r}\right)^{2} \approx-\pi R_{0} \delta \rho v^{*} \frac{R_{0}^{2} \omega^{2}(t)}{\delta^{2}} .
$$

On the other hand, the kinetic energy (per unit length) of the accelerated solid-body rotation of the vortex core is determined by the expression

$$
E_{k i n}(t)=\pi \rho \int_{0}^{R_{0}} r d r \cdot v_{\varphi}^{2}(r, t)=\frac{\pi}{4} \rho R_{0}^{4} \cdot \omega^{2}(t) .
$$

In this case, the growth rate of the kinetic energy of vortex rotation accelerated under the action of the convective and Coriolis hydrodynamic forces is

$$
\frac{d E_{k i n}}{d t}=\frac{\pi}{2} \alpha \rho R_{0}^{4} \cdot \omega(t) \frac{d \omega}{d t} \approx \frac{\pi}{2} \alpha \rho R_{0}^{4} \cdot \omega^{2}(t) .
$$

Comparing expressions (29) and (31), we obtain the following condition for the weak dissipation of the vortex energy: $v^{*}<\alpha \cdot R_{0} \cdot \delta / 2$. For the above parameter values, $v^{*} \approx 8 \cdot 10^{7} \mathrm{~m}^{2} / \mathrm{s}, \alpha \approx 8 \cdot 10^{-3} \mathrm{~s}^{-1}, R_{0} \approx 500 \mathrm{~km}$, and $\delta \approx 25 \mathrm{~km}$, the left- and the right-hand sides of this inequality are nearly equal to one another, i.e., the rate of kinetic energy dissipation is nearly equal to the energy gain caused by the convective and Coriolis forces. As a result, in the final stage of evolution under the condition of strong turbulence in the surface layer, the MHD vortex reaches a regime of steady-state rotation and gradually dissipates.

There is another energy dissipation mechanism in an MHD vortex that is related to ohmic losses leading to Joule heating. The heat is generated by the electric currents induced by the growing magnetic fields that flow in plasma with a finite conductivity. According to Maxwell's equations

$$
\operatorname{rot} \mathbf{H}=\frac{4 \pi}{c} \cdot \mathbf{j}, \quad \operatorname{rot} \mathbf{E}=-\frac{1}{c} \frac{\partial \mathbf{H}}{\partial t}
$$

the generation of magnetic fields during the exponential evolution of MHD vortices should be accompanied by the generation of electric currents $\mathbf{j}$ and electric fields $\mathbf{E}$, which are related to one another via Ohm's law $\mathbf{j}=\sigma \mathbf{E}$. However, taking into account that the vortex evolution is a relatively slow process, we can consider only the first of Maxwell's equations. In particular, the axial magnetic field, which is uniform inside the vortex core and vanishes 
in the transition layer of thickness $\delta$, induces the azimuthal current

$$
j_{\varphi}(t)=-\frac{c}{4 \pi} \cdot \frac{\partial H_{z}(t)}{\partial r} \approx-\frac{c \cdot h(t)}{4 \pi \delta} .
$$

in this layer.

The azimuthal current of density (33) that flows inside the turbulent surface layer should lead to heat release in this layer, the power of which per unit vortex length is

$$
Q \approx 2 \pi R_{0} \delta \cdot j_{\varphi}^{2} / \sigma=c^{2} R_{0} \cdot h^{2}(t) / 8 \pi \delta \sigma .
$$

Here $\sigma=e^{2} n_{e} \cdot \tau_{e} / m_{e}$ is the electron conductivity of the chromospheric plasma with the electron density $n_{e} \approx 3 \cdot 10^{15} \mathrm{~cm}^{-3}, m_{e}$ is the mass of an electron, and $\tau_{e} \approx 10^{-13} \mathrm{~s}$ is the average time of electron-electron and electron-ion Coulomb collisions at an electron temperature of $T_{e} \approx 10^{4} \mathrm{~K}$. Hence, in the order of magnitude, we have $\sigma \approx 10^{9} \mathrm{~s}^{-1}$.

As a result, setting $h(t) \approx h(0) \cdot \exp \left(\alpha t_{0}\right)$, we find that the Joule heating power in the final stage of MHD vortex evolution is $Q \approx 1 \mathrm{MJ} / \mathrm{km}$.

Joule losses must slow down the MHD vortex rotation, determined by relationships (38). The slowing down will be weak if

$$
\frac{d E_{k i n}(t)}{d t}=\frac{\pi}{2} \alpha \rho R_{0}^{4} \cdot \omega^{2}(t) \gg Q(t) .
$$

Taking into account that the angular velocity $\omega(t)$ and the longitudinal magnetic field $h(t)$ grow according to the same exponential law, we can rewrite inequality (43) in the form

$$
\frac{c^{2} \cdot c_{A}^{2}(0)}{\pi \alpha \cdot \delta \cdot \sigma \cdot R_{0} \cdot V_{0}^{2}} \ll 1,
$$

which shows that, for the above parameter values, $\alpha \approx 8 \cdot 10^{-3} \mathrm{~s}^{-1}, \quad \sigma \approx 10^{9} \mathrm{~s}^{-1}, \quad \delta \approx 25 \mathrm{~km}, \quad$ and $R_{0} \approx 500 \mathrm{~km}$, the Joule losses weakly affect MHD vortex dynamics if $V_{0}>c_{A 0}$.

Due to ohmic heat release in the MHD vortex shell, additional heating and ionization of the chromospheric plasma, followed by its recombination upon cooling, take place. This should lead to the glow of MHD vortices in the visible and UV spectral regions. Since MHD vortices can reach the lower layers of the solar corona, heat released due to the ohmic heating of plasma, along with that released due to the dissipation of the magnetic and kinetic energies of vortices during their decay, can play a significant role in the heating of the solar corona to temperatures as high as $10^{6} \mathrm{~K}$. However, this issue requires additional study, which goes beyond the scope of the present work.
To conclude, it should be emphasized that the initial local vorticity of the solar plasma in the regions where MHD vortices begin to form can have opposite signs, so that the vortices in different solar hemispheres can rotate in different directions. Accordingly, the self-consistent axial magnetic fields generated by such vortices should have opposite directions. Thus it can be energetically advantageous for the magnetic field lines of the adjacent pairs of such vortices and anti-vortices to be closed into loops, which are indeed often observed in solar atmosphere.

Acknowledgements. The author is grateful to A.B. Mikhailovskii, N.S. Erokhin, D.D. Sokolov, and A.V. Stepanov for useful discussions of the problems touched upon in this work, as well as V.I. Pentegov for his help in preparing this manuscript for publication.

\section{References}

1. Wedermeyer-Böhm S., Scullion E., Steiner O. et al.: 2012, Nature, 486, 505.

2. Wedermeyer-Böhm S., van der Voort L. R.: 2009, Astron. Astrophys., 507.

3. De Pontieu B., McIntosh S. W., Carlsson M., et al.: 2007, Science, 318, 1574.

4. Cirtain J. W., Golub L., Lundquist L., et al.: 2007, Science, 318, 1580.

5. Lemen J. R., Title A. M., Akin D. J., et al.: 2012, Sol. Phys., 275, 17.

6. McIntosh S. W., De Pontieu B., Carlsson M., et al.: 2011, Nature, 475, 477.

7. Fedun V., Shelyag S., Verth G., et al.: 2011, Ann. Geophys., 29, 1029.

8. Van Ballegooijen A., Asgari-Targhi M., Cranmer S., DeLuca E.: 2011, Astrophys. J., 736, 3.

9. Kitiashvili I., Kosovichev A., Mansour N., Wray A.: 2011, Astrophys. J., 727, L50.

10. Attie R., Innes D., Potts H.: 2009, Astron. Astrophys., 493, L13.

11. Shelyag S., Keys P., Mathioudakis M., Keenan F. P.: 2011, Astron. Astrophys., 526, A5.

12. Landau L. D., Lifshitz E. M.: Electrodynamics of Continuous Media (Fizmatgiz, Moscow, 1959; Pergamon, Oxford, 1960).

13. Pashitskii E. A.: 2010, JETP, 110, 1026.

14. Balmaceda L., Domingues S. V., Palacios J., et al.: 2010, Astron. Astrophys., 513.

15. Zhang J., Liu Y.: 2011, Astrophys. J., 741, L7. 